\title{
Molecular epidemiology and the clinical impact of carbapenemase-producing Enterobacterales isolates among adult patients: aspects from a Romanian non-teaching hospital
}

\author{
Annamária Földes ${ }^{1,2 *}$, Szabolcs Molnár ${ }^{3}$, Doina-Veronica Bilca ${ }^{4}$, \\ Septimiu Toader Voidăzan ${ }^{5}$, Edit Székely,
}

1. Department of Microbiology, Laboratory of Medical Analysis, "Dr. Constantin Opriş" County Emergency Hospital of Baia Mare, Romania

2. PhD student, George Emil Palade University of Medicine, Pharmacy, Science, and Technology of Targu Mures, Romania

3. PhD student, Department of Microbiology, George Emil Palade University of Medicine, Pharmacy, Science, and Technology of Targu Mures, Romania

4. Department of Microbiology, Central Clinical Laboratory, Targu Mures County Emergency Clinical Hospital, Romania

5. Department of Epidemiology, George Emil Palade University of Medicine, Pharmacy,

Science, and Technology of Targu Mures, Romania

6. Department of Microbiology, George Emil Palade University of Medicine, Pharmacy,

Science, and Technology of Targu Mures, Romania

\begin{abstract}
Introduction: A dramatic increase of infections induced by carbapenemase-producing Enterobacterales (CPE) has been registered worldwide. The aim of this study was to evaluate the molecular epidemiology and the clinical impact of CPE strains isolated from adult inpatients. Material and methods: A one-year, single-center, retrospective observational study including 34 consecutive patients with 37 non-duplicate CPE strains recovered from clinical specimens was accomplished. The Vitek 2 Compact, M.I.C.Evaluator strips, the modified carbapenem inactivation method (mCIM), and the combination disks test (KPC, MBL, OXA-48 Confirm kit, Rosco Diagnostica) were applied as phenotypic tests. A multiplex polymerase chain reaction (PCR) assay was used for detection of blaKPC, blaNDM, and blaOXA-48-like genes. The clonality was assessed with pulsed-field gel electrophoresis (PFGE). Results: Klebsiella pneumoniae $(n=25)$ was the most frequent CPE encountered. The carbapenemase types were $\operatorname{NDM}(n=13), K P C(n=12)$, and OXA-48-like (n=12). Two distinct clonal clusters were identified among the 12
\end{abstract}

\footnotetext{
* Corresponding author: Annamária Főldes, Department of Microbiology, Laboratory of Medical Analysis, ”Dr. Constantin Opriş" County Emergency Hospital of Baia Mare, Romania. E-mail: anafoldes@gmail.com
} 
KPC positive strains. All CPE isolates exhibited non-susceptibility to carbapenems, cephalosporins, ciprofloxacin. Respiratory tract infections $(n=16)$ and hospitalization in the intensive care unit (ICU) $(n=14)$ were dominant. The most common comorbidity was congestive heart failure $(n=11)$. Monotherapy was the main strategy adopted $(n=15)$. Death occurred in 18 patients. Conclusions: Our analysis underscores the scarcity of antibiotic solutions and high mortality. Monotherapy for urinary tract infections (UTIS) is beneficial. Inter- or intrahospital dissemination of successful epidemic clones is proved. The adequate CPE infections control programs and antimicrobial policies are essential..

Keywords: carbapenemases, Enterobacterales, antimicrobial treatment, mortality

Received: $16^{\text {th }}$ July 2020; Accepted: $11^{\text {th }}$ October 2020; Published: $16^{\text {th }}$ October 2020

\section{Introduction}

The members of the Enterobacterales order (formerly Enterobacteriaceae) are Gram-negative enteric rod-shaped bacteria responsible for a wide variety of human infections in both healthy and compromised hosts $(1,2)$.

The increasing antimicrobial resistance in these bacteria and emergence of new infectious syndromes have evolved as a global public health crisis in recent years (2-4). The therapeutic approach of these multidrug-resistant (MDR) and extensively drug-resistant (XDR) infections remains problematic generally because of the escalation of carbapenem resistance (2-5), which has been recently reconfirmed as an urgent public health threat by the United States Centers for Disease Control and Prevention (CDC) (6).

The primary mechanism of carbapenem resistance remains the acquisition of various carbapenemases which variably inactivate carbapenems and other members of the beta-lactam antimicrobial class $(2,3,7-9)$. These enzymes are encoded on large transferable plasmids which are able to rapidly and widely spread and often coexpress linked resistance to fluoroquinolones, aminoglycosides, trimetoprim-sulfamethoxazole, and tetracyclines $(2-4,8,10)$.

The prevention of CPE infections and optimization of therapeutic regimens are challenging and require early and accurate detection techniques of CPE strains $(7,10)$.
In 2018 the European Centre for Disease Prevention and Control (ECDC) alerted about the emergence of resistance of $\mathrm{CPE}$ isolates to ceftazidime-avibactam, a newly authorized drug with activity especially against Klebsiella pneumoniae carbapenemase (KPC) producers (11). Some large Romanian medical institutions have confirmed the presence and dissemination of different types of carbapenemases (12-21), but the involvement of risk factors, clinical impact of CPE pathogens, treatment, and patients' outcomes have not been sufficiently explored. Furthermore, a Romanian national centralized database of circulating carbapenemase types is not yet available.

In this context, the present study investigated the molecular epidemiology and the clinical impact of CPE strains isolated from hospitalized patients to obtain a more comprehensive picture of occurrence, spread, clinical characteristics, and antimicrobial treatment. The premise of this study was that there is no difference among the patients' groups diagnosed with New Delhi metallo- $\beta$-lactamase (NDM), KPC, and oxacillinase-48-like (OXA-48-like) carbapenemases.

\section{Material and methods}

Ethical Approval was granted by the Ethics Committees of the "Dr. Constantin Opriş" County Emergency Hospital Baia Mare, Romania (ref- 
erence number 14598/04.06.2019) and George Emil Palade University of Medicine, Pharmacy, Science, and Technology of Targu Mures, Romania (reference number 405/11.10.2019).

\section{Setting, study design and participants}

A single-center, retrospective observational study of all inpatients microbiologically documented with CPE isolates from clinical specimens was conducted in the "Dr. Constantin Opriș" County Emergency Hospital Baia Mare, Romania from $1^{\text {st }}$ of January to $31^{\text {st }}$ of December 2017. This is a public 920-bed general acute care non-teaching hospital with emergency, intensive care units, surgical, and medical wards.

All consecutive non-duplicate CPE strains isolated from clinical samples were included. Diverse species or even the same species recovered from the same patient were taken into account if they carried different carbapenemase genes. Recurrent CPE infections with the same species harbouring the same gene encoding carbapenem-hydrolysing enzyme isolated from the same anatomical site and diagnosed in the previous 12 months were excluded.

\section{Data collection and definitions}

The descriptive analysis included patients' medical records review. The following details were recorded: demographics, date of admission, ward, previous healthcare services, microbiological characteristics, coexisting medical conditions, clinical, laboratory, and imaging findings, invasive procedures, exposure to possible predisposing factors, treatment, and outcomes. Empiric treatment was considered as any antimicrobial drug potentially active against aerobic Gram-negative bacilli administered for approximately 3 calendar days from the specimen collection date to the date of available susceptibility test results for CPE pathogen. Active empiric antibiotic regimen included at least 1 agent with documented in vitro sensitivity. Targeted thera- py was defined as sensitivity-adjusted treatment that started on or at least 3 calendar days after the date on which the complete antibiotic susceptibility profile was obtained. Carbapenems were defined as active agents if the minimum inhibitory concentration (MIC) was $\leq 8 \mathrm{mg} / \mathrm{L}$.

Follow-up cultures negative for CPE pathogens were interpreted as microbiological eradication. The 30 day all-cause mortality was measured starting from the date of the first positive CPE culture collection.

\section{Bacterial identification and antimicrobial sus- ceptibility profile}

The microbiological diagnosis and the antibiotic susceptibility tests were based on standard procedures, Vitek 2 Compact (bioMérieux, France), API 20E (bioMérieux, France), and accompanied in some cases by M.I.C.Evaluator strip tests for meropenem (Oxoid, Thermo Fisher Scientific, UK). The results were interpreted consonant with the Clinical and Laboratory Standards Institute (CLSI) standard 2017 (22).

\section{Phenotypic and molecular analysis}

The mCIM $(22,23)$ and the combination disks test were applied for all carbapenem non-susceptible strains belonging to the Enterobacterales order. The strains were frozen at $-70^{\circ} \mathrm{C}$, subcultured on solid medium, and then a multiplex PCR method for the identification of carbapenemase-encoding genes (bla ${ }_{\mathrm{KPC}}$, bla ${ }_{\mathrm{NDM}}$, and blaoxA-48-like) was performed (12).

\section{Molecular typing}

The PFGE was achieved according to a CDC Pulsenet protocol (24) for all KPC-producing $K$. pneumoniae isolates and all bla ${ }_{\mathrm{NDM}}$ positive $P$. stuartii strains. Total bacterial genome was digested with the Xbal restriction endonuclease (ThermoFisher, USA) in case of $K$. pneumoniae strains and NotI restriction endonuclease (ThermoFisher, USA) in case of $P$. stuartii isolates. 
The resultant macrorestriction fragments were separated by electrophoresis on a CHEF-DR III system (Bio-Rad Laboratories, USA). The PFGE patterns were analysed according to Tenover criteria (25).

\section{Statistical analysis}

The statistical protocol for quantitative and qualitative data was parsed. Descriptive summary statistics and the Kolmogorov-Smirnov test for assessing the normality of distribution were used for numerical data. For continuous variables we used one-way ANOVA or Kruskal-Wallis test. For categorical data, differences were analyzed using the Chi-squared test for trend.

The level of significance was set at $\mathrm{a}=0.05$.

For statistical calculations, GraphPad 3.6 State Software, San Diego, California, USA, was used.

\section{Results}

\section{Microbiological characteristics of clinical iso- lates}

During the assessment period, a total of 2412 consecutive strains belonging to the order Enterobacterales were identified, with E. coli $(\mathrm{n}=1397 ; 57.91 \%)$, Klebsiella spp. $\quad(\mathrm{n}=423$; $17.53 \%)$, Proteus spp. ( $\mathrm{n}=233 ; 9.66 \%)$, Enterobacter spp. ( $\mathrm{n}=159 ; 6.59 \%)$, Morganella spp. $(\mathrm{n}=82 ; 3.39 \%)$, Citrobacter $\mathrm{spp} .(\mathrm{n}=56 ; 2.32 \%)$, Serratia spp. $(\mathrm{n}=38 ; 1.57 \%)$, and Providencia spp. ( $\mathrm{n}=19 ; 0.78 \%)$ being the most important species. Of these, 92 (3.81\%) carbapenem non-susceptible isolates were detected, and only $37(1.53 \%)$ non-duplicate strains recovered from 34 inpatients were phenotypically confirmed as CPE producers.

The distribution of CPE isolates was as follows: K. pneumoniae $(\mathrm{n}=25 ; 67.56 \%)$, Serratia $\mathrm{spp}$. $(\mathrm{n}=6 ; 16.21 \%)$, E. coli $(\mathrm{n}=2 ; 5.40 \%)$, P. stuartii $(\mathrm{n}=2 ; 5.40 \%), M$. morganii $(\mathrm{n}=1 ; 2.70 \%)$, and $E$. cloacae complex $(\mathrm{n}=1 ; 2.70 \%)$.

\section{Diversity of carbapenem-hydrolysing enzymes}

The carbapenemase types determined phenotypically by the combination disks test were distributed as follows: NDM ( $\mathrm{n}=13 ; 35.13 \%)$, $\operatorname{KPC}(\mathrm{n}=12 ; 32.43 \%)$, and OXA-48-like $(\mathrm{n}=12$; $32.43 \%$ ). Multiplex PCR analysis confirmed carbapenemase-encoding genes in 35 available strains with complete consensus with the MCIM and the combination disks test results. All MBL strains carried $b l a_{\mathrm{NDM}}$ genes. No strain harbored multiple carbapenemase genes.

\section{PFGE typing}

The analysis of PFGE fingerprints of all 12 KPC positive strains illustrated that they belonged to 2 different clonal clusters (A and B) exhibiting more than 6 band differences (Figure 1). The dominant cluster A consisted of 10 isolates with almost the same similarity level of relatedness, except for a variation of one additional band observed only in 2 strains. Seven out of the 10 patients with isolates included in cluster A were previously admitted to other Romanian hospitals, 4 of them in the same surgical unit of the same university hospital. The two strains with one additional band were isolated from patients who previously received medical treatment in two different Romanian university hospitals approximately 60 days apart between the strains' isolation date in our laboratory. Cluster B included 2 genetically indistinguishable isolates recovered from blood cultures collected from patients with haematological malignancies hospitalized in the same ward of our hospital two months apart.

The PFGE restriction patterns of 2 bla $_{\mathrm{NDM}}$ positive $P$. stuartii isolates were similar.

\section{Relation to hospitalization in other Romanian hospitals and identification of multiple CPE isolates in the same patient}

One year prior detection of CPE strains in our laboratory, 12 out of the 34 patients were previ- 


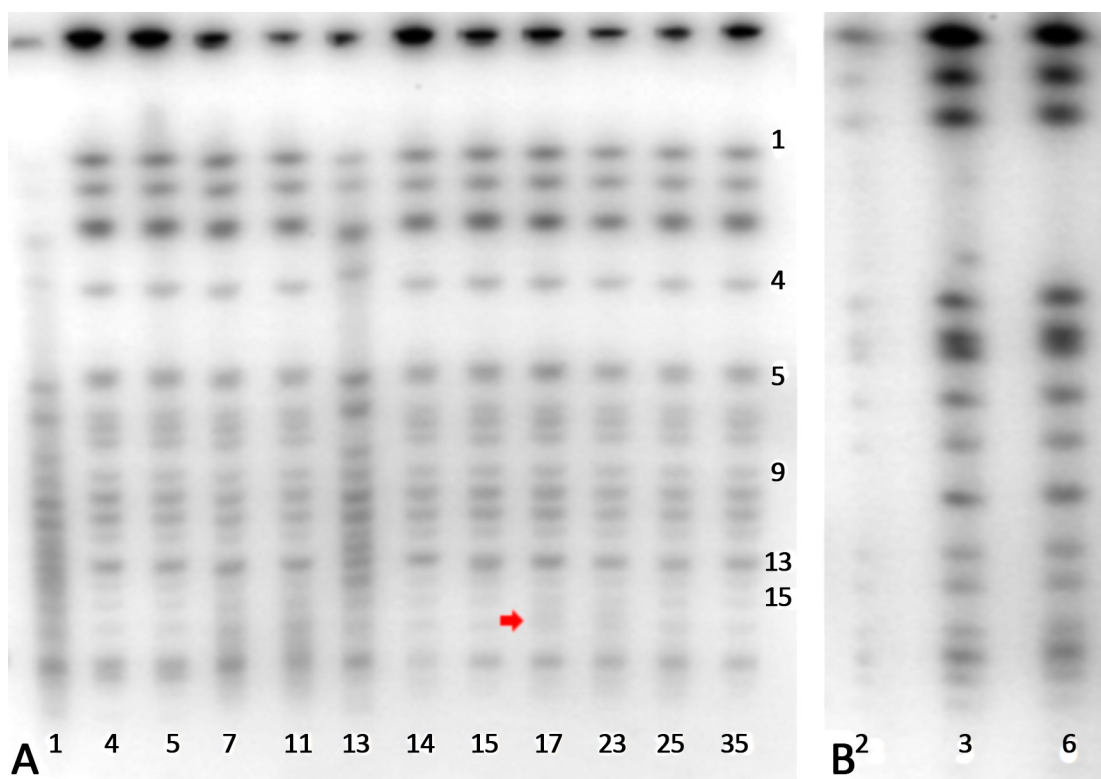

Figure 1. The PFGE patterns for 12 bla $_{\mathrm{KPC}}$ K. pneumoniae strains (1A) and for 2 bla $_{\mathrm{NDM}}$ P. stuartii isolates (1B). 1A: Isolates No. 1 and 13 belong to the cluster B, and the rest to the cluster A. Isolates No. 17 and 23 with one additional band (red arrow). 1B: Isolates No. 3 and 6 are CPE and isolate No. 2 is a $P$. stuartii non-CPE

ously admitted to other Romanian hospitals, 10 of them to university hospitals located in other geographical regions. No history of healthcare exposure abroad was noted.

Two patients from the same ICU room yielded bla $a_{\mathrm{NDM}}$ positive $P$. stuartii and $b l a_{\mathrm{KPC}}$ positive $K$. pneumoniae isolates in consecutive endotracheal aspirates identified within 2 weeks, one of the patients being previously hospitalized in a university hospital. One patient had $b l a_{\text {OXA-48-like }}$ positive E. coli and $K$. pneumoniae strains simultaneously present in the same wound sample.

\section{Antimicrobial susceptibility profile of CPE producers}

All CPE strains were MDR and showed 100\% non-susceptibility to meropenem, imipenem, ertapenem, aminopenicillins, cephalosporins, and ciprofloxacin (Table 1). The MIC to meropenem was performed in 28 out of the total of 37 strains and a value $\leq 8 \mathrm{mg} / \mathrm{L}$ was noted exclusively in 8 OXA-48-like producers.
Susceptibility to tigecycline was noted in 22 out of the 24 strains tested. Colistin displayed in vitro activity against 13 out of the $21 \mathrm{CPE}$ tested strains of K. pneumoniae and E. coli. Fosfomycin was active antimicrobial agent against 6 out of the 7 urinary CPE strains tested.

\section{Clinical and epidemiological characteristics}

A total of 34 patients were diagnosed with infections, 19 male and 15 female. Their mean age was 59 years (range $30-86$ years). The CPE strains were recovered from respiratory tract specimens $(\mathrm{n}=16 ; 43.24 \%)$, urine $(\mathrm{n}=10 ; 27.02 \%)$, wounds $(\mathrm{n}=8 ; 21.62 \%)$, and blood $(\mathrm{n}=3 ; 8.10 \%)$ collected from patients hospitalized in the intensive care unit (ICU) ( $\mathrm{n}=14 ; 41.17 \%)$, surgical units $(\mathrm{n}=13$; $38.23 \%$ ), and medical wards ( $\mathrm{n}=7 ; 20.58 \%$ ).

The median period of hospitalization of all patients before CPE detection was 3 days (range $0-46$ days). Twenty-two out of the 34 patients $(64.70 \%)$ were hospitalized for more than 48 hours before the collection date of the first positive CPE clinical specimen. The remaining 12 


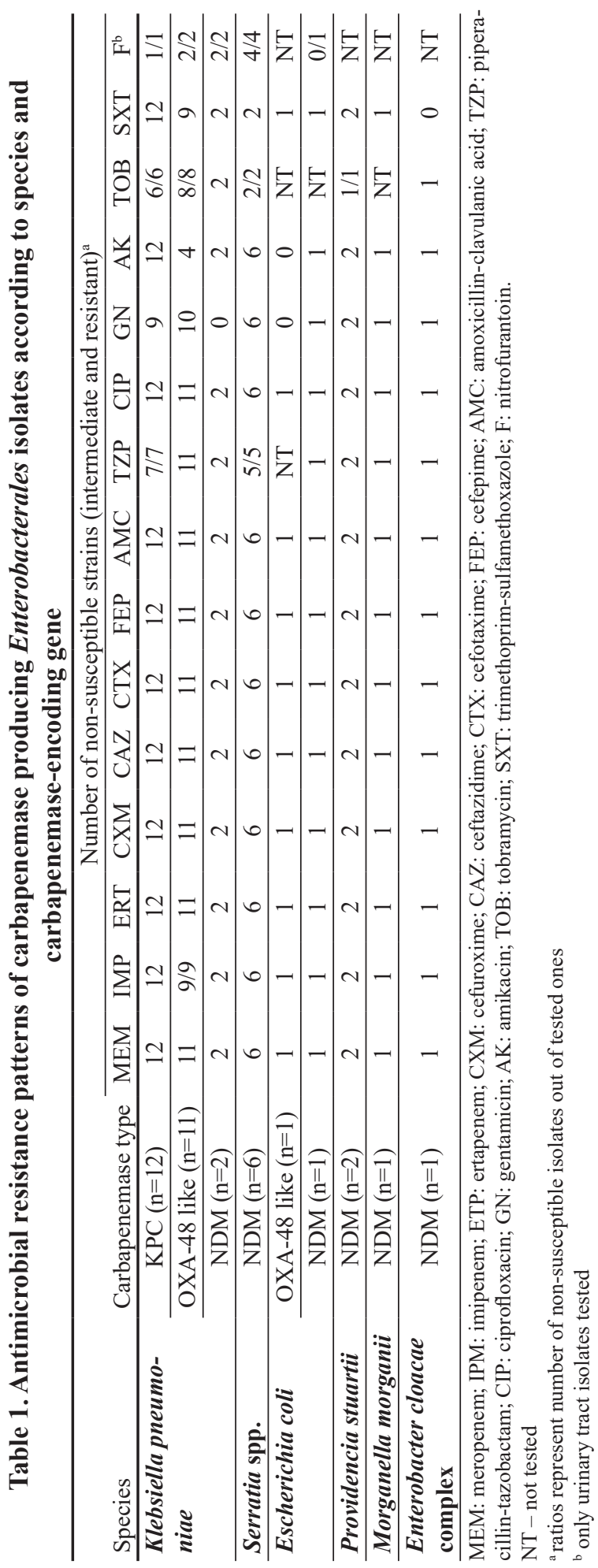

patients (35.29\%) developed CPE infections within 48 hours from hospital admission, but they had received healthcare services during the previous year in different Romanian hospitals.

The study participants were subjects to various potential risk factors. No statistically significant differences were found among the three patient groups in terms of demographic and medical characteristics (Table 2).

All of our participants presented in their medical records symptoms and/or signs of infection, or had a clinical diagnosis of infection stated by the treating physician. Seven out of the 34 patients (20.58\%) were diagnosed with sepsis.

The occurrence of coinfections was noted in 18 out of the total patients, 8 of them being microbiologically documented with Acinetobacter baumannii complex.

CPE screening on admission was performed only in few patients and a prior CPE positive screening sample was documented in 6 patients.

\section{Antimicrobial treatment}

Most of our participants $(\mathrm{n}=30)$ received systemic empirical treatment with antimicrobial drugs, mainly cephalosporins, fluoroquinolones, $\beta$-lactam/ $\beta$-lactamase inhibitor combinations, and aminoglycosides (Table 3). A statistically significant difference was noted between OXA-48like patients compared to NDM and KPC groups for the active empirical treatment (P 0.005).

In agreement with the susceptibility profiles of the CPE pathogens, 19 out of the total patients were treated with targeted antibiotic regimens, especially as monotherapy $(n=15)$ with colistin, tigecycline, trimethoprim-sulfamethoxazole, aminoglycosides, or fosfomycin trometamol. Seven patients with urinary CPE isolates received monotherapy. The combination therapy with active drugs selected in 3 of our patients included tigecycline with aminoglycosides, or colistin with amikacin, while in 1 septic patient with bla OXA-48-like positive $K$. pneumoniae strain 
Table 2. Demographic characteristics of patients with CPE infections and underlying medical conditions*

\begin{tabular}{|c|c|c|c|c|c|}
\hline Demographics and medical conditions of patients & $\begin{array}{l}\text { All patients } \\
\quad(n=34)\end{array}$ & $\begin{array}{c}\text { NDM } \\
\text { strains } \\
(n=12) *\end{array}$ & $\begin{array}{c}\text { KPC } \\
\text { strains } \\
(n=11) *\end{array}$ & $\begin{array}{c}\text { OXA-48-like } \\
\text { strains } \\
(\mathrm{n}=11)^{*}\end{array}$ & P value \\
\hline Male & 19 & 7 & 4 & 8 & 0.64 \\
\hline Female & 15 & 5 & 7 & 3 & 0.56 \\
\hline Age** (mean $\pm \mathrm{SD}) ;$ (range); years & $\begin{array}{c}59 \pm 13.5(30 \\
-86) \\
\end{array}$ & $\begin{array}{l}59 \pm 14.3 \\
(39-74) \\
\end{array}$ & $\begin{array}{l}58 \pm 14.9 \\
(30-75) \\
\end{array}$ & $\begin{array}{r}62 \pm 11.9 \\
(44-86) \\
\end{array}$ & 0.92 \\
\hline \multicolumn{6}{|l|}{ Source of isolate } \\
\hline Respiratory tract & 14 & 3 & 5 & 6 & 0.62 \\
\hline Urinary tract & 10 & 7 & 1 & 2 & 0.13 \\
\hline Wounds & 7 & 2 & 2 & 3 & 0.86 \\
\hline Bloodstream & 3 & 0 & 3 & 0 & 0.06 \\
\hline Body temperature $* *\left({ }^{\circ} \mathrm{C}\right)($ mean $\pm \mathrm{SD}) ;$ (range) & $\begin{array}{c}37.2 \pm 0.9 \\
(35.7-39.6) \\
\end{array}$ & $\begin{array}{c}37.1 \pm 0.6 \\
(36.5-38.4) \\
\end{array}$ & $\begin{array}{c}37.5 \pm 1.0 \\
(36.2-39.6) \\
\end{array}$ & $\begin{array}{c}37.1 \pm 1.0 \\
(35.7-39.0) \\
\end{array}$ & 0.52 \\
\hline \multicolumn{6}{|l|}{ Patient-specific risk factors } \\
\hline Leucocyte count $(/ \mu \mathrm{L})<1.000$ & 3 & 1 & 2 & 0 & 0.38 \\
\hline $1.000-4.000$ & 1 & 0 & 0 & 1 & 0.37 \\
\hline $4.000-10.000$ & 15 & 8 & 1 & 6 & 0.14 \\
\hline$>10.000$ & 15 & 3 & 8 & 4 & 0.35 \\
\hline Thrombocyte count $(/ \mu \mathrm{L})<150.000$ & 7 & 0 & 3 & 4 & 0.16 \\
\hline 1 year prior healthcare & 29 & 10 & 9 & 10 & 0.98 \\
\hline Transfer from another hospital & 12 & 4 & 5 & 3 & 0.82 \\
\hline $\begin{array}{l}\text { Days from hospital admission to collecting positive } \\
\text { clinical sample*** (IQR) }\end{array}$ & $3(0-46)$ & $3(0-33)$ & $3(0-25)$ & $9(0-46)$ & 0.78 \\
\hline 3 months prior use of broad spectrum antibiotics & 27 & 9 & 10 & 8 & 0.92 \\
\hline Previous surgical interventions & 21 & 8 & 6 & 7 & 0.95 \\
\hline Urinary catheters & 21 & 9 & 5 & 7 & 0.77 \\
\hline Previous ICU stay & 19 & 5 & 6 & 8 & 0.73 \\
\hline Mechanical ventilation & 19 & 5 & 7 & 7 & 0.79 \\
\hline Central venous/arterial catheters & 18 & 5 & 7 & 6 & 0.83 \\
\hline Immunosuppressive treatment including steroids & 15 & 4 & 5 & 6 & 0.81 \\
\hline Transplant recipient & 0 & 0 & 0 & 0 & NA \\
\hline Parenteral nutrition & 15 & 4 & 5 & 6 & 0.81 \\
\hline Chemotherapy & 6 & 2 & 3 & 1 & 0.64 \\
\hline Hormone therapy & 3 & 1 & 1 & 1 & 0.99 \\
\hline Radiotherapy & 3 & 0 & 2 & 1 & 0.37 \\
\hline Coinfections & 18 & 7 & 5 & 6 & 0.94 \\
\hline \multicolumn{6}{|l|}{ Comorbidities } \\
\hline Congestive heart failure & 11 & 2 & 5 & 4 & 0.54 \\
\hline Chronic renal failure & 9 & 3 & 1 & 5 & 0.33 \\
\hline Neuropsychological disorders & 9 & 2 & 5 & 2 & 0.44 \\
\hline Solid neoplasm & 8 & 4 & 2 & 2 & 0.74 \\
\hline Diabetes mellitus & 6 & 1 & 3 & 2 & 0.60 \\
\hline Traumatisms and burns & 6 & 4 & 1 & 1 & 0.35 \\
\hline Hematologic malignancies & 3 & 1 & 2 & 0 & 0.38 \\
\hline Dialysis & 2 & 1 & 0 & 1 & 0.62 \\
\hline Microbiological eradication & 8 & 3 & 2 & 3 & 0.91 \\
\hline 30-day mortality**** & $18 / 31$ & $3 / 10$ & $8 / 10$ & 7 & 0.46 \\
\hline
\end{tabular}

*Only the first clinical isolate per patient and 1 strain per patient in case of coinfection with 2 OXA-48-like strains were included. Data are shown as number of patients, media**, or median***. IQR: the interquartile range.

****Three patients had no available 30-day mortality data. NA: not applicable 
Table 3. Therapeutic regimens in patients with CPE infections*

\begin{tabular}{lccccc}
\hline Treatment & $\begin{array}{c}\text { All patients } \\
(\mathbf{n}=\mathbf{3 4})\end{array}$ & $\begin{array}{c}\text { NDM strains } \\
(\mathbf{n}=\mathbf{1 2}) *\end{array}$ & $\begin{array}{c}\text { KPC strains } \\
(\mathbf{n}=\mathbf{1 1}) *\end{array}$ & $\begin{array}{c}\text { OXA-48-like } \\
\text { strains (n=11)* }\end{array}$ & P value \\
\hline Empirical treatment & 30 & 9 & 10 & 11 & 0.89 \\
\hline Active empirical treatment & 10 & 1 & 0 & 9 & $\mathbf{0 . 0 0 5}$ \\
\hline Meropenem & 3 & 0 & 0 & 3 & 0.06 \\
\hline Colistin & 4 & 1 & 0 & 3 & 0.20 \\
\hline Aminoglycosides & 1 & 0 & 0 & 1 & 0.37 \\
\hline Fosfomycin trometamol & 1 & 0 & 0 & 1 & 0.37 \\
\hline Trimethoprim-sulphamethoxazole & 1 & 0 & 0 & 1 & 0.37 \\
\hline Tigecycline & 0 & 0 & 0 & 0 & NA \\
\hline Targeted treatment & 19 & 7 & 4 & 8 & 0.64 \\
\hline
\end{tabular}

*Only the first clinical isolate per patient and 1 strain per patient in case of coinfection with 2 OXA-48-like strains were included. Data are shown as number of patients. NA: not applicable

which exhibited meropenem $\mathrm{MIC} \leq 8 \mathrm{mg} / \mathrm{L}$ the therapeutic scheme comprised of meropenem and amikacin. The targeted antibiotic therapy was not administered in 14 patients, half of them deceased before microbiological results became available.

\section{Outcomes}

Following therapeutic interventions, including targeted antibiotic treatment, endotracheal tubes, or ureteral stent replacements, the microbiological eradication of CPE pathogens was achieved in 8 cases $(23.52 \%)$, but only 4 of these survived by 30 days. Two patients with positive CPE endotracheal aspirate specimens and associated infections developed microbiological eradication without an available targeted antibiotic treatment. Overall, 18 out of the 31 participants with available survival data (58.06\%) died within 30 days of the first positive CPE specimen collection. Of the seven septic patients five deceased and one was transferred to another hospital unit without the possibility to follow-up. Despite administration of targeted antimicrobial treatment approximately half of the patients with available follow-up data $(n=8)$ did not survive by 30 days. Five out of the 7 participants with urinary CPE isolates treated with monotherapy had a favourable outcome.

\section{Discussion}

This investigation highlighted the phenotypic, genotypic, epidemiological, and clinical features of CPE pathogens isolated in 2017 from adult patients admitted to a non-teaching hospital.

The majority of our CPE isolates were $K$. pneumoniae $(\mathrm{n}=25)$, a key pathogen responsible for the most of the worldwide CPE infections, especially healthcare-related ones. Consistent with other reports $(26,27)$, all significant carbapenemase-encoding genes were demonstrated in our K. pneumoniae isolates. Attributable mortality for carbapenem resistant $K$. pneumoniae rose six-fold between 2007-2015 (27). In 2018, 25$50 \%$ of the invasive $K$. pneumoniae isolates reported by Romanian hospitals to the European Antimicrobial Resistance Surveillance Network (EARS-Net) presented resistance to carbapenems. These data might not be representative, since our national population coverage was under $11 \%(27)$.

Interestingly, our data revealed an approximately similar distribution among the three main types of carbapenemases: NDM ( $\mathrm{n}=13), \mathrm{KPC}(\mathrm{n}=12)$, and OXA-48-like $(n=12)$. Previous Romanian publications described a considerable variety of detected carbapenemases in different medical institutions, OXA-48-like or NDM producers were 
the most frequently encountered (12-17, 19-21). The present study did not find any strain harbouring multiple carbapenemase-encoding genes. In contrast, Germany reported recently a first outbreak of K. pneumoniae sequence type (ST) 307 as a worldwide emerging high-risk clone, which co-produced NDM-1 and OXA-48, and exhibited colistin resistance (28).

Regarding our PFGE findings, 10 KPC producing $K$. pneumoniae isolates belonging to the principal cluster A illustrated interhospital dissemination of a successful epidemic K. pneumoniae clone, and the rest of 2 strains affiliated to the cluster B indicated a possibile intrahospital spread. Our 2 bla $_{\mathrm{NDM}}$ positive $P$. stuartii strains belonged to a successful $P$. stuartii clone which disseminated nationwide as reported recently by Molnar et al. (18). Another Romanian study described a similar expansion of a $K$. pneumoniae OXA-48 positive clone (14). A permanent interaction between expansion of high-risk clones and plasmid-mediated resistance genes transmission is contributing to the continuous global CPE epidemic $(4,10)$.

In agreement with several studies $(3-5,7,8,11$, 28-30), our CPE strains expressed MDR and XDR phenotypes with limited treatment options, such as aminoglycosides, colistin, tigecycline, trimethoprim-sulfamethoxazole, fosfomycin, and nitrofurantoin. Most of our tested strains preserved susceptibility to tygecycline, but development of resistance during therapy with this drug has already been documented (31). Consistent with other observations some of our KPC and OXA-48-like producers were susceptible to gentamicin and amikacin $(3,7)$.

Colistin resistance was noted in less than half of our K. pneumoniae and E. coli tested strains. An increasing number of hospital outbreaks due to colistin-resistant CPE isolates, especially Klebsiella spp. have been reported globally $(3,4,7$, $8,28,29$ ), and both chromosomal and recently, transferable plasmid-mediated genes have been involved $(3,4,27,32)$. Determination of colistin susceptibility is technically problematic, and the reference method for testing is broth microdilution $(27,32)$.

The highest level of antimicrobial drug resistance was observed in $b l a_{\mathrm{NDM}}$ positive $P$. stuartii and M. morganii isolates, as previously outlined by Molnar et al (18).

To the best of our knowledge this is the first Romanian research attempting to investigate the notable risk factors, clinical data, outcomes, and treatment for CPE isolates.

In our analysis, most of the infections $(\mathrm{n}=22)$ were hospital-acquired (with clinical onset more than 48 hours after hospital admission), and the remaining $(\mathrm{n}=12)$ were healthcare-associated, as described elsewhere (33).

Prior the first CPE detection the median duration of hospitalization of our participants was 3 days (range 0-46 days) (P 0.78). In Canada, Kohler et al. reported a median of 2.5 days from admission to diagnosis of CPE strains, for inpatients (P 0.03) (34).

Several risk factors associated with CPE colonization or infection assessed in our study have also been mentioned by other authors $(10,35)$. Prior hospitalization, use of broad spectrum antibiotics, surgical interventions, urinary catheters, admission to ICU, mechanical ventilation, and indwelling central venous or arterial catheters were all identifiable risk factors in our patients. All of our participants presented symptoms or sign of infections. Overall, half of them $(n=18)$ experienced concurrent infections, most often with Acinetobacter baumannii complex, and in two of these, microbiological eradication of CPE pathogens occurred in the absence of targeted antimicrobial treatment. This aspect could be explained by the fact that the last two subjects had endotracheal tube colonization with CPE strains. Additionally, the studied population presented diverse comorbid conditions. This target population is frequently not included in the design of 
clinical trials for registration of new antimicrobial agents (36).

Despite the escalating burden of CPE infections and the ample published information, data regarding patient's outcome and treatment guidelines for CPE infections have a low level of scientific quality of evidence, based mainly on limited retrospective observational studies or case series $(3,8,29,30,36,37)$. Disappointingly, randomized controlled trials in this topic are insufficient and some of them without statistical power $(3,29)$.

The management of CPE infections is challenging and consists of administration of older or newly approved antimicrobial compounds, dose modifications, and combination therapy schemes including or not a carbapenem $(3,8)$. The treatment should be individualized in compliance with the anatomical site of infection, severity of disease, comorbid conditions, susceptibility results for all potentially in vitro active agents and the available drugs $(3,29)$.

Our research revealed a statistically significant association between the active empirical treatment administered and the dependent variable (P 0.005). This aspect should be interpreted with caution since more than half of our patients with OXA-48-like producers died $(n=7)$. However, a multicentre study did not find statistically significant differences between the empiric antimicrobial therapy used for CPE infections and the patients' outcomes (36).

The targeted treatment strategy adopted for the majority of our patients was monotherapy $(n=15)$, and only in 4 cases a double combination regimen was applied. Mainly observational studies found a survival benefit in high-risk patients with septic shock, or bloodstream infections when a combination treatment with at least 2 agents was used $(3,4,29)$, while monotherapy would be more suitable for lower-risk subjects (3). None of our patients received carbapenem as a single active agent for treating carbapenem intermediate-susceptible strains. The efficacy of carbapenems in monotherapy for these infections is unreliable due to the lack of controlled clinical studies $(3,22)$. Out of the total of our CPE strains, only 8 OXA-48-like producers expressed meropenem MIC under $8 \mathrm{mg} / \mathrm{L}$, and in one of these patients with intra-abdominal infection and sepsis a dual targeted therapy with meropenem and amikacin was administred. In septic shock induced principally by KPC producers with meropenem $\mathrm{MIC} \leq 8 \mathrm{mg} / \mathrm{L}$ and in the absence of new agents such as ceftazidim-avibactam, meropenem-vaborbactam, or other available in vitro active antibiotics according to the primary site of infection, administration of high-doses of meropenem in extended infusion in association with another active agent could be an attractive solution, but this aspect cannot be extrapolated to OXA-48-like or MBL producers $(3,29)$. Overall, the double treatment regimen consists of various combinations including colistin, carbapenems, tigecycline, aminoglycosides, and fosfomycin (3, 4, 8, 37). Promising new antimicrobial agents for treating CPE infections are imipenem/cilastatin-relebactam, plazomycin, eravacycline, cefiderocol, and aztreonam-avibactam $(4,29)$.

Our high rate of 30-day all-cause mortality $(58.06 \%)$ was similar to previous data that indicated mortality caused by serious CPE infections ranging from 30 to $70 \%$ (38). In contrast, another study mentioned a mortality of $16 \%$, but more than $80 \%$ of the cases were UTIs (34).

\section{Limitations of the study}

The retrospective nature of this single-center investigation and the limited number of occurrences of these cases do not allow us to generalize our results for all Romanian hospitals. The PFGE analysis and follow-up cultures were not undertaken for all cases. Surveillance cultures for CPE pathogens were performed only in some patients. 


\section{Conclusions}

The current study emphasizes the scarcity of antibiotic solutions and high mortality. Some of our K. pneumoniae and E. coli strains exhibit resistance to colistin. Monotherapy for UTIs due to CPE pathogens is beneficial. Fosfomycin is a potential therapeutic agent for urinary isolates. The MICs testing by standardized methods is important. Establishing the clinical impact of the CPE isolates and the optimal treatment strategy, especially in patients with several comorbidities and coinfections are fundamental. All of our cases are hospital- or healthcare-associated, therefore, adequate $\mathrm{CPE}$ infection control programs and antimicrobial policies are essential for limiting their spread.

In the future, more studies regarding our national distribution of CPE isolates, identification of high-risk clones, mechanisms of antibiotic resistance, and clinical correlations including the new antimicrobial agents are needed.

\section{Authors' contributions}

Conceived, designed the research: AF, ES. Investigated, analyzed the data: AF, ES, SM. Formal analysis: STV, AF. Wrote the manuscript: AF. Critically revised the paper: ES, SM, AF, STV, DVB.

\section{Conflicts of Interest}

No potential conflicts of interest relevant to this article were reported. This study was not funded.

\section{References}

1. Adeolu M, Alnajar S, Naushad S, Gupta RS. Genome-based phylogeny and taxonomy of the 'Enterobacteriales': proposal for Enterobacterales ord. nov.divided into the families Enterobacteriaceae, Erwiniaceae fam. nov., Pectobacteriaceae fam. nov., Yersiniaceae fam. nov., Hafniaceae fam. nov., Morganellaceae fam. nov., and Budviciaceae fam. nov. Int J Syst Evol Micr.
2016;66:5575-99. DOI: 10.1099/ijsem.0.001485

2. Russo TA, Johnson JR. Diseases caused by Gram-negative enteric bacilli. In: Kasper DL, Fauci AS, Hauser SL, Longo DL, Jameson JL, Loscalzo J, editors. Harrison's infectious diseases, 3rd edition. USA: McGraw Hill Education 2017;58:507-20.

3. Rodriguez-Bano J, Gutierrez-Gutierrez B, Machuca I, Pascuala A. Treatment of infections caused by extended-spectrum-beta-lactamase-, AmpC-, and carbapenemase-producing Enterobacteriaceae. Clin Microbiol Rev. 2018;31(2):e00079-17. DOI: 10.1128/ CMR.00079-17

4. European Centre for Disease Prevention and Control. Rapid Risk Assessment. Carbapenem-resistant Enterobacteriaceae, second update, 26 September 2019. Stockholm: ECDC; 2019. Available from: https://www. ecdc.europa.eu/sites/default/files/documents/carbapenem-resistant-enterobacteriaceae-risk-assessment-rev-2. pdf, accessed 24th May 2020.

5. Magiorakos AP, Srinivasan A, Carey RB, Carmeli Y, Falagas ME, Giske CG et al. Multidrug-resistant, extensively drug-resistant and pandrug-resistant bacteria: an international expert proposal for interim standard definitions for acquired resistance. Clin Microbiol Infect. 2012;18:268-81. DOI: 10.1111/j.14690691.2011.03570.x

6. The United States Centers for Disease Control and Prevention. Antibiotic resistance threats in the United States 2019 (2019 AR Threats Report), Atlanta: CDC; 2019. Available from: https:/www.cdc.gov/drugresistance/pdf/threats-report/2019-ar-threats-report-508. pdf, accessed 24th May 2020.

7. Cui X, Zhang, Du H. Carbapenemases in Enterobacteriaceae: detection and antimicrobial therapy. Front Microbiol. 2019;10:1823. DOI: 10.3389/fmicb.2019.01823

8. Morrill HJ, Pogue JM, Kaye KS, LaPlante KL. Treatment options for carbapenem-resistant Enterobacteriaceae infections. Open Forum Infect Dis. 2015;2(2):ofv050. DOI: 10.1093/ofid/ofv050

9. Bonomo RA, Burd EM, Conly J, Limbago BM, Poirel L, Segre JA et al. Carbapenemase-producing organisms: a global scourge. Clin Infect Dis. 2018;66:12907. DOI: $10.1093 / \mathrm{cid} / \mathrm{cix} 893$

10. van Duin D, Doi Y. The global epidemiolo $\neg$ gy of carbapenemase-producing Enterobacte-riaceae. Virulence 2017;8(4):460-9. DOI: $10.1080 / 21505594.2016 .1222343$ 
11. European Centre for Disease Prevention and Control. Rapid Risk Assessment. Emergence of resistance to ceftazidime-avibactam in carbapenem-resistant Enterobacteriaceae, 12 June 2018. Stockholm: ECDC; 2018. Available from:https://www.ecdc.europa.eu/sites/default/files/documents/RRA-Emergence-of-resistanceto $\% 20 \mathrm{CAZ}$-AVI-in-CRE-Enterobacteriaceae.pdf, accessed 24th May 2020.

12. Szekely E, Damjanova I, Janvari L, Vas KE, Molnar $\mathrm{S}$, Bilca DV, et al. First description of blaNDM-1, blaOXA-48, blaOXA-181 producing Enterobacteriaceae strains in Romania. Int $\mathrm{J}$ of Med Microbiol. 2013;303(8):697-700. DOI: 10.1016/j. ijmm.2013.10.001

13. Braun SD, Dorneanu OS, Vremera T, Reissig A, Monecke S, Ehricht R. Carbapenemase-producing Enterobacteriaceae: a 2-year surveillance in a hospital in Iaşi, Romania. Future Microbiol. 2016;11(3):391-401. DOI: $10.2217 / \mathrm{fmb} .15 .148$

14. Lixandru BE, Cotar AI, Straut M, Usein CR, Cristea D, Ciontea $\mathrm{S}$ et al. Carbapenemase-producing Klebsiella pneumoniae in Romania: A Six-Month Survey. PLoS One. 2015;10:e0143214. DOI: 10.1371/journal. pone.0143214

15. Gheorghe I, Czobor I, Chifiriuc MC, Borcan E, Ghita $\mathrm{C}$, Banu $\mathrm{O}$ et al. Molecular screening of carbapenemase-producing Gram-negative strains in Romanian intensive care units during a one year survey. J Med Microbiol. 2014;63:1303-10. DOI: 10.1099/ jmm.0.074039-0

16. Rafila A, Talapan D, Dorobat OM, Popescu GA, Pitigoi D, Florea D et al. Emergence of carbapenemase-producing Enterobacteriaceae, a public health threat: a Romanian Infectious Disease Hospital based study. Rev Romana Med Lab. 2015;23:295-301. DOI: 10.1515/ rrlm-2015-0024

17. Foldes A, Bilca DV, Szekely E. Phenotypic and molecular identification of carbapenemase-producing Enterobacteriaceae - challenges in diagnosis and treatment. Rev Romana Med Lab. 2018;26:221-30. DOI: 10.2478/ rrlm-2018-0018

18. Molnar S, Flonta MMM, Almas A, Buzea M, Licker M, Rus $M$ et al. Dissemination of NDM-1 carbapenemase-producer Providencia stuartii strains in Romanian hospitals: a multicentre study. J Hosp Infect. 2019;103:165-9. DOI: 10.1016/j.jhin.2019.04.015

19. Dortet L, Flonta M, Boudehen YM, Creton E, Ber- nabeu S, Vogel A et al. Dissemination of carbapenemase producing Enterobacteriaceae and Pseudomonas aeruginosa in Romania. Antimicrob Agents Chemother 2015;59(11):7100-3. DOI: 10.1128/AAC.01512-15

20. Popescu C, Popescu GA, Dorobat O, Rafila A, Tanase D, Mikula C, et al. OXA-48-carbapene $\neg$ mase-producing Klebsiella pneumoniae infections - the first cases diagnosed in Romanian National Institute of Infectious Diseases. Rev Romana Med Lab. 2017;25(1):55-61. DOI: $10.1515 / \mathrm{rrlm}-2017-0006$

21. Predoiu G, Muntean MM, Muntean A, Popa MI, Sandu IT, Aurelian J et al. Phenotypic and molecular detection of carbapenemase-producing gram negative bacilli in a tertiary urology hospital. EurUrolSuppl. 2018;17(12):e2736. DOI: 10.1016/S15699056(18)33573-5

22. Performance Standards for antimicrobial susceptibility testing, 27th informational supplement. CLSI document M100-S27. Clinical and Laboratory Standards Insti $\neg$ tute, 2017.

23. van der Zwaluw K, de Haan A, Pluister GN, Bootsma HJ, de Neeling AJ, Schouls LM - The carbapenem inactivation method (CIM), a simple and low-cost alternative for the Carba NP Test to assess phenotypic carbapenemase activity in Gram-negative rods. PLoS ONE. 2015;10(3):1-13. e0123690. DOI: 10.1371/journal.pone. 0123690

24. Standard Operating Procedure for PulseNet PFGE of Escherichia coli O157:H7, Escherichia coli non-O157 (STEC), Salmonella serotypes, Shigella sonnei and Shigella flexneri. Available from: https:/www.cdc. gov/pulsenet/pdf/ecoli-shigella-salmonella-pfge-protocol-508c.pdf, accessed 25th May 2020.

25. Tenover FC, Arbeit RD, Goering RV, Mickelsen PA, Murray BE, Persing DH et al. Interpreting chromosomal DNA restriction patterns produced by Pulsed-Field Gel Electrophoresis: criteria for bacterial strain typing. J Clin Microbiol. 1995:33(9):2233-9. DOI: 10.1128/ JCM.33.9.2233-2239.1995

26. World Health Organization. Global antimicrobial resistance surveillance system (GLASS) report: early implementation 2016-2017. Geneva: WHO; 2017. Available from:https://apps.who.int/iris/bitstream/handle/10665 /259744/9789241513449-eng.pdf;jsessionid=F157BC737B4E5899BE88D4F414129581? sequence=1, accessed 26th May 2020.

27. European Centre for Disease Prevention and Control. 
Surveillance Report. Surveillance of antimicrobial resistance in Europe 2018. Stockholm: ECDC; 2019. Available from: https://www.ecdc.europa.eu/sites/default/files/documents/surveillance-antimicrobial-resistance-Europe-2018.pdf, accessed 26th May 2020.

28. European Centre for Disease Prevention and Control. Outbreak of carbapenemase-producing (NDM-1 and OXA-48) and colistin-resistant Klebsiellapneumoniae ST307, north-east Germany, 2019. 28 October 2019. Stockholm: ECDC; 2019. Available from: https://www. ecdc.europa.eu/sites/default/files/documents/Klebsiella-pneumoniae-resistance-Germany-risk-assessment. pdf, accessed 29th May 2020.

29. Durante-Mangoni E, Andini R, Zampino R. Management of carbapenem-resistant Enterobacteriaceae infections. Clin Microbiol. Infect 2019;25:943-50. DOI: 10.1016/j.cmi.2019.04.013

30. Jacobs DM, Courtney Safir M, Huang D, Minhaj F, Parker A, Rao GG. Triple combination antibiotic therapy for carbapenemase-producing Klebsiella pneumoniae: a systematic review. Ann Clin Microbiol Antimicrob. 2017;16(1):76. DOI: 10.1186/s12941-017-0249-2

31. Du X, He F, Shi Q, Zhao F, Xu J, Fu Y et al. The rapid emergence of tigecycline resistance in blaKPC2 harboring Klebsiella pneumoniae, as mediated in vivo by mutation in tetA during tigecycline treatment. Front Microbiol. 2018;9:648. DOI: 10.3389/fmicb.2018.00648

32. Jayol A, Nordmann P, Lehours P, Poirel L, Dubois V. Comparison of methods for detection of plasmid-mediated and chromosomally encoded colistin resistance in Enterobacteriaceae. Clin Microbiol Infect. 2018;24:175-9. DOI: 10.1016/j.cmi.2017.06.002

33. Cardoso T, Almeida M, Friedman ND, Aragao I,
Costa-Pereira A, Sarmento AE et al. Classification of healthcare-associated infection: a systematic review 10 years after the first proposal. BMC Med. 2014;12:40. DOI: 10.1186/1741-7015-12-40

34. Kohler PP, Melano RG, Patel SN, Shafinaz S, Faheem A, Coleman BL et al. Emergence of carbapenemase-producing Enterobacteriaceae, South-Central Ontario, Canada. Emerg Infect Dis. 2018;24(9):167482. DOI: $10.3201 /$ eid2409.180164

35. Safdar N, Maki DG. The commonality of risk factors for nosocomial colonization and infection with antimicrobial-resistant Staphylococcus aureus, Enterococcus, Gram-negative bacilli, Clostridium difficile, and Candida. Ann Intern Med. 2002;136:834-44. DOI: 10.7326/0003-4819-136-11-200206040-00013

36. Alexander EL, Loutit J, Tumbarello M, Wunderink R, Felton T, Daikos G et al. Carbapenem-resistant Enterobacteriaceae infections: results from a retrospective series and implications for the design of prospective clinical trials. Open Forum Infect Dis. 2017;4(2):ofx063. DOI: 10.1093/ofid/ofx063

37. Martin A, Fahrbach K, Zhao Q, Lodise T. Association between carbapenem resistance and mortality among adult, hospitalized patients with serious infections due to Enterobacteriaceae: results of a systematic literature review and meta-analysis. Open Forum Infect Dis. 2018;5(7):ofy150. DOI: 10.1093/ofid/ofy150

38. Tischendorf J, Almeida de Avila R, Safdar N. Risk of infection following colonization with carbapenem-resistant Enterobactericeae: A systematic review. Am J Infect Control. 2016;44(5):539-43. DOI: 10.1016/j. ajic.2015.12.005 